\title{
Ressourcen von Grundschullehrkräften in inklusiven Settings und ihr Zusammenspiel mit inklusiver Selbstwirksamkeit
}

\author{
Birte Oetjen · Sabine Martschinke • Christian Elting • \\ Rebecca Baumann · Lars Wissenbach
}

Eingegangen: 30. Oktober 2020 / Angenommen: 1. April 2021 / Online publiziert: 9. Juli 2021 (C) Der/die Autor(en) 2021

Zusammenfassung Die vorliegende Studie untersucht Ressourcen in inklusiven Settings und prüft ihr Zusammenspiel mit inklusiver Selbstwirksamkeit. In einem Mixed-Methods-Design wurden 43 Grundschullehrkräfte in Interviews nach ihren persönlichen Ressourcen in inklusiven Settings gefragt. Flankierend wurde in einem Online-Fragebogen inklusive Selbstwirksamkeit standardisiert erfasst. Die Ergebnisse zeigen, dass Grundschullehrkräfte besonders häufig soziale Unterstützung und Selbstregulation, weniger Überzeugungen, Werthaltungen und Vertrauen in die eigenen Fähigkeiten oder pädagogisch-didaktisches Wissen als Ressourcen beschreiben. Als potenziell handlungsrelevante Variable wird die inklusive Selbstwirksamkeit zu einem bedeutenden Anteil von sozialer Unterstützung und Selbstregulation erklärt.

Schlüsselwörter Ressourcen in inklusiven Settings · Inklusive Selbstwirksamkeit · Umgang mit Heterogenität · Lehrkräftegesundheit

\footnotetext{
Birte Oetjen ( $₫)$. Prof. Dr. Sabine Martschinke · Christian Elting · Rebecca Baumann · Lars Wissenbach Institut für Grundschulforschung, Friedrich-Alexander-Universität Erlangen-Nürnberg, Regensburger Str. 160, 90478 Nürnberg, Deutschland

E-Mail: birte.oetjen@fau.de

Prof. Dr. Sabine Martschinke

E-Mail: sabine.martschinke@fau.de

Christian Elting

E-Mail: christian.elting@fau.de

Rebecca Baumann

E-Mail: rebecca.baumann@fau.de

Lars Wissenbach

E-Mail: lars.wissenbach@fau.de
} 


\title{
Resources of primary school teachers in inclusive education and their interplay with teachers' inclusive self-efficacy
}

\begin{abstract}
The present study examines resources in inclusive education as well as the interplay of these with teachers' self-efficacy to implement inclusive practices. The study is based on a mixed methods design, which included interviews with 43 primary teachers on their individual resources and an online self-efficacy questionnaire. Overall, the results show that teachers describe the use of social support and selfregulated strategies the most, whereas attitudes and the trust in oneself competence and pedagogical and didactic knowledge were barely mentioned. Moreover, teachers' self-efficacy to implement inclusive practices, which is a promising variable to predict teachers' actions in the classroom, was predicted by the use of social support and self-regulation.
\end{abstract}

Keywords Resources in inclusive education - Self-efficacy to implement inclusive practices $\cdot$ Dealing with/managing heterogeneity · Teachers' health

\section{Problemaufriss}

Mit der Ratifizierung der UN-Behindertenrechtskonvention von 2009 sowie Migrations- und Fluchtbewegungen erweitert sich die Heterogenität der Schülerschaft stetig. Dabei hat Inklusion, im weiten Sinn als erfolgreicher und produktiver Umgang mit Heterogenität in all ihren Facetten (König et al. 2019), zu einer Veränderung der Arbeitstätigkeit von Lehrkräften geführt (Peperkorn et al. 2020). Erste empirische Studien zeigen, dass durch eine „heterogenisierte“ Schülerschaft und Kinder mit (sonder-)pädagogischem Förderbedarf berufliche Anforderungen wachsen (Martschinke et al. 2020) und mit negativem Beanspruchungserleben seitens der Lehrkräfte verknüpft sein können (Hedderich 2016). Auch zeigen Studien ungünstige Effekte eines negativen Beanspruchungserlebens auf die Unterrichtsgestaltung von Lehrkräften und die Leistungen von Schülerinnen und Schülern (Klusmann und Richter 2014). Vor diesem Hintergrund erscheint es von zentraler Bedeutung, Ressourcen von Lehrkräften in inklusiven Settings zu identifizieren, die ungünstiges Beanspruchungserleben reduzieren können (Rothland und Klusmann 2016). Die hier vorgestellte Studie untersucht Ressourcen von Grundschullehrkräften in inklusiven Settings und prüft Zusammenhänge zur inklusiven Selbstwirksamkeit. Selbstwirksamkeitserwartung als ,subjektive Gewissheit, neue oder schwierige Anforderungssituationen aufgrund der eigenen Kompetenz bewältigen zu können“ (Schwarzer und Warner 2014, S. 662) bezieht sich in inklusiven Settings auf die Zuversicht einer Lehrkraft, inklusiven Unterricht selbst gestalten zu können (Bosse et al. 2017). Neben ihrer Bedeutung als Ressource können Selbstwirksamkeitserwartungen auch prädiktiv für Unterrichtspraxis und -qualität sein (Kiel et al. 2020). 


\section{Theoretischer und empirischer Hintergrund}

Aufgaben und Bedingungen einer beruflichen Tätigkeit stellen objektive Belastungen (Rudow 1994) oder Anforderungen (Cramer et al. 2018) dar, welche als Faktoren ,unabhängig vom Individuum existieren und potenziell Beanspruchungen hervorrufen“ (Rudow 1994, S. 42). Subjektive Belastungen entstehen durch die Widerspiegelung objektiver Belastungsfaktoren und können zu positiven und negativen Beanspruchungsreaktionen (kurzfristig) und -folgen (langfristig) führen (Rudow 1994). Ressourcen moderieren in diesem Zusammenhang die Verarbeitung beruflicher Anforderungen (Cramer et al. 2018; Stiller 2015). Eine Erklärung, wie Arbeitsanforderungen und Arbeitsressourcen positive (z.B. Arbeitszufriedenheit) und negative Beanspruchungsfolgen (z. B. Burnout) beeinflussen, liefert das Job-Demands-Resources-Modell (Bakker und Demerouti 2007). Im Rahmen des Modells konnte auch für den Lehrberuf gezeigt werden, dass sich Arbeitsressourcen günstig auf negatives Beanspruchungserleben auswirken können (Dicke et al. 2018).

\subsection{Anforderungen in inklusiven Settings}

In inklusiven Settings beziehen sich Anforderungen allgemein auf inklusionsbezogene pädagogische Tätigkeiten (Peperkorn et al. 2020). Lindner und Schwab (2020) zählen hierzu Kollaboration und Co-Teaching, Maßnahmen der Binnendifferenzierung, Anpassungen der didaktischen Vermittlungsstrategie oder des Materials, Feedback sowie die individuelle Unterstützung. Einem weiten Inklusionsverständnis folgend zielen diese Tätigkeiten auf die passgenaue Förderung der Leistungsund Persönlichkeitsentwicklung aller Kinder gemäß ihren individuellen Förderbedarfen ab (Elting et al. 2019) und stellen Lehrkräfte somit vor die Anforderung, allen Kindern bestmöglich gerecht zu werden.

Neben diesen allgemeinen Anforderungen geben Studien der Lehrerbelastungsforschung sowie erste Studien in inklusiven Settings (im weiten Sinn) auch Hinweise auf mögliche spezifischere Anforderungen. So zählt das Unterrichten von schwierigen, störenden sowie verhaltensauffälligen Schülerinnen und Schülern zu einem der meistgenannten Belastungsfaktoren von Lehrkräften (Krause et al. 2013; Kyriacou 2001). Eine erste Systematisierung, welche Merkmale von Kindern Grundschullehrkräfte als Anforderung wahrnehmen, liefert eine Interviewstudie von Martschinke et al. (2020). Die Forschungsgruppe identifizierte fünf Merkmale von Kindern, wobei sich das am häufigsten genannte Merkmal auf Probleme durch informell beobachtete oder formal attestierte (sonder-)pädagogische Förderbedarfe und deren Feststellung bezieht. Weitere in dieser Studie identifizierte Fallmerkmale sind problematische Familienhintergründe, sozial-emotionale Probleme, störendes Unterrichts- und Arbeitsverhalten sowie Lern- und Leistungsprobleme. Die Lehrkräfte schreiben den Kindern dabei nicht allein ein Merkmal zu, sondern komplexe, intersektional verwobene Merkmalsmuster, die von ihnen als herausfordernd wahrgenommen werden (ebd.). Eine weitere Anforderung stellt das Unterrichten in heterogen zusammengesetzten Klassen dar (Bennemann 2019).

Per se ist die Umsetzung von Inklusion mit erweiterten Anforderungen durch einzelne Kinder oder durch die Heterogenität von Klassen allerdings noch nicht mit 
einem ausschließlich negativen Beanspruchungserleben verknüpft, da die subjektive Bewertung von Anforderungen entscheidend ist (Rudow 1994) und potenziell auch positive Beanspruchungsfolgen auftreten können (Bakker und Demerouti 2007).

\subsection{Ressourcen in inklusiven Settings}

Negative Beanspruchungsreaktionen und -folgen treten dann in Erscheinung, ,wenn die Belastungen angesichts der zur Verfügung stehenden Ressourcen zu einer deutlichen Über- oder Unterforderung führen" (Rothland und Klusmann 2016, S. 355). Ob Anforderungen zu positiven oder negativen Beanspruchungsreaktionen und -folgen führen, hängt somit von der Verfügbarkeit und dem Ausmaß vorhandener Ressourcen ab (Bakker und Demerouti 2007; Stiller 2015). Ressourcen, als Handlungsvoraussetzungen psychologischer, sozialer oder auch organisatorischer Art (Bakker und Demerouti 2007; Rothland und Klusmann 2016), können als personexterne (z.B. Rahmenbedingungen, soziale Netzwerke) oder personinterne (z. B. Selbstregulation, Einstellung) Ressourcen vorliegen (Cramer et al. 2018; Peperkorn et al. 2020). Neben günstigen Rahmenbedingungen konnten Studien insbesondere soziale Unterstützung sowie Aspekte der professionellen Kompetenz von Lehrkräften (Baumert und Kunter 2011; Rothland 2013a) als wichtige Ressourcen identifizieren (Rothland und Klusmann 2016; Schaarschmidt 2005).

Soziale Unterstützung gilt dabei als eine der bedeutendsten Arbeitsressourcen im Lehrberuf (Rothland 2013b) und bezeichnet ,die tatsächlich (erhaltene) oder die erwartete (wahrgenommene) Hilfsinteraktion zwischen einem Unterstützungsgeber und einem Unterstützungsempfänger" (Kienle et al. 2006, S. 109f.). Erste empirische Arbeiten zur Bewältigung von Anforderungen in inklusiven Settings unterstreichen die Bedeutsamkeit sozialer Unterstützung (Bennemann 2019; Saloviita und Takala 2010) und identifizieren als wichtige Quellen das Kollegium, die Schulleitung, zusätzliches pädagogisches Personal, das eigene private Umfeld sowie die unterrichteten Schülerinnen und Schüler (Bennemann 2019).

Für die erfolgreiche Bewältigung beruflicher Anforderungen sind zudem Professionswissen, selbstregulative Fähigkeiten, Überzeugungen und Werthaltungen sowie motivationale Orientierungen als Aspekte professioneller Kompetenz bedeutsam (Rothland 2013a). Deklaratives, prozedurales und strategisches Professionswissen bildet dabei den Kern professioneller Kompetenz (Baumert und Kunter 2011). Studien zum Belastungs- und Beanspruchungserleben von Lehrkräften schreiben besonders dem allgemeinen pädagogisch-psychologischen Wissen eine moderierende Wirkung zu (Dicke et al. 2015; Wesselborg et al. 2014). Dieses Wissen bezieht sich auf Lernen und Lernende, auf den Umgang mit der Klasse als komplexes soziales Gefüge, auf methodisches Repertoire sowie auf die Gestaltung von Lernumgebungen (Voss et al. 2015).

Wenn mit Inklusion erweiterte Anforderungen verknüpft sind, scheint es auf der personinternen Ebene notwendig, auch mit negativen Emotionen umgehen und mit seiner Energie haushalten zu können, d.h. selbstregulative Fähigkeiten erfolgreich einzusetzen (Baumert und Kunter 2011; Schaarschmidt und Kieschke 2013). Dazu gehört zum einen eine akzeptierende Haltung den eigenen Erfahrungen und Empfindungen gegenüber sowie Distanzierungsfähigkeit (Schaarschmidt 2005). Zum an- 
deren sind die Fertigkeit und Fähigkeit, eigene Emotionen zu regulieren, bewusst wahrzunehmen und auszudrücken sowie Aktivitäten, die der Erholung dienen (Lehr 2014), bedeutsam. Speziell für inklusive Settings liegen bislang nur erste Befunde vor, die jedoch auf die Bedeutsamkeit von selbstregulierenden Strategien und Maßnahmen verweisen (Bennemann 2019).

Im inklusiven Kontext sind auch Überzeugungen und Werthaltungen sowie motivationale Orientierungen vielversprechend (Baumert und Kunter 2011). Inklusive Einstellungen als Teil von Überzeugungen richten sich auf personimmanente Bewertungen, ob das gemeinsame Unterrichten einer heterogenen Schülerschaft befürwortet oder abgelehnt wird (Bosse et al. 2017). Demgegenüber beschreiben inklusionsbezogene Selbstwirksamkeitserwartungen (je nach Modellierung unterschiedlich, oftmals jedoch den motivationalen Orientierungen zugeordnet; s. Kap. 6), inwieweit Lehrkräfte ihre Tätigkeit im inklusiven Kontext für bewältigbar halten (Bosse et al. 2017; Hecht et al. 2016). Sowohl in als auch außerhalb inklusiver Settings kennzeichnet überforderte oder resignierte Lehrkräfte dabei eine geringe Selbstwirksamkeit (Abele und Candova 2007; Urton et al. 2014). Dahingegen weisen Lehrkräfte mit hoher Selbstwirksamkeit ein reduziertes negatives Beanspruchungserleben auf (Abele und Candova 2007; Wudy und Jerusalem 2011) und nehmen Unterrichtsstörungen als weniger beanspruchend wahr (Dicke et al. 2018). Spezifisch für inklusive Settings gibt es jedoch ein erstes gegenläufiges Ergebnis, das weiteren Forschungsbedarf aufdeckt: In einer Studie von Bosse et al. (2016) konnten nur inklusive Einstellungen ungünstiges Beanspruchungserleben vorhersagen, nicht aber die Selbstwirksamkeit.

\subsection{Zur Bedeutung von Selbstwirksamkeit für unterrichtliches Handeln}

Schwarzer und Warner (2014) merken an, dass eine hoch ausgeprägte Selbstwirksamkeitserwartung ,den einzelnen Lehrer nicht nur vor gesundheitlichen Problemen [...] bewahren kann, sondern darüber hinaus Lehrer- sowie Schülerverhalten im Unterricht positiv beeinflussen [...] kann“ (S. 666). Demnach sind Selbstwirksamkeitserwartungen eine wichtige Voraussetzung für erfolgreiche Handlungsregulation im Unterricht (Schwarzer und Warner 2014). Somit kommt der (inklusiven) Selbstwirksamkeit neben ihrer Bedeutung als Ressource auch eine handlungsregulierende Funktion zu (Bandura 1977; Urton et al. 2014). So geben Lehrkräfte mit hohen Selbstwirksamkeitserwartungen z. B. an, mehr differenzierende Unterrichtsstrategien anzuwenden oder anwenden zu wollen (Kiel et al. 2020; Lübke et al. 2016). Zu ähnlichen Ergebnissen kommen Studien mit Lehramtsstudierenden: Bei geringen Selbstwirksamkeitserwartungen werden instruktive Lehr-Lernformate im inklusiven Setting präferiert (Knigge und Rotter 2015). Dass die Wahl offener Unterrichtsformen, die flexible Unterrichtsgestaltung sowie die Schülerorientierung u.a. vom Ausmaß der Selbstwirksamkeit abhängt, konnten auch Döring-Seipel und Dauber (2010) nachweisen. Mehrere Studien belegen zudem, dass eine erhöhte Selbstwirksamkeit mit qualitativ hochwertigem Unterrichtshandeln einhergeht (Klassen und Tze 2014) und sowohl mit Kriterien guten Unterrichts als auch mit Schülerleistung positiv verknüpft ist (Fauth et al. 2019). Befunde zum Zusammenhang inklusiver Selbstwirksamkeit als handlungsregulierende Variable und Ressourcen, welche die 
Lehrkräfte in inklusiven Settings als hilfreich erachten, konnten bislang nicht ausgemacht werden.

\section{Modellannahme und Fragestellungen}

Der bisherige Forschungsstand zeigt, dass besonders soziale Unterstützung sowie Aspekte der professionellen Kompetenz wichtig für die erfolgreiche Bewältigung beruflicher Anforderungen sind. Erste Studien in inklusiven Settings im engen Sinn unterstreichen dies. Allerdings stehen derzeit Studien aus, die ein weites Inklusionsverständnis zugrunde legen und vor diesem Hintergrund Ressourcen von Grundschullehrkräften differenziert beschreiben. Dabei besteht insbesondere Forschungsbedarf im Bereich der nicht-kognitiven, affektiv-motivationalen Aspekte professioneller Kompetenz (Rothland 2013a, S. 11f). Neben divergierenden Befunden zum Beitrag dieser Ressourcen zum Beanspruchungserleben ist auch inhaltlich noch wenig darüber bekannt, welche dieser Ressourcen Grundschullehrkräfte in inklusiven Settings als hilfreich erachten. Dementsprechend werden in dieser Untersuchung als bedeutsame personinterne Ressourcen für die erfolgreiche Bewältigung von Anforderungen im inklusiven Setting Überzeugungen und Werthaltungen (inklusive Einstellungen), motivationale Orientierungen (inklusive Selbstwirksamkeitserwartungen) und Selbstregulation sowie als personexterne Ressource soziale Unterstützung deduktiv gesetzt.

Ferner sind Effekte der Ressourcennutzung in inklusiven Settings, z. B. auf die Unterrichtsgestaltung, weitgehend ungeklärt. In diesem Beitrag wird die Modellannahme geprüft, dass die in der Wahrnehmung der Lehrkräfte hilfreichen Ressourcen interindividuelle Unterschiede in der standardisiert erfassten inklusiven Selbstwirksamkeit erklären können. Über eine Triangulation qualitativer und quantitativer Daten können hier lediglich erste Hinweise erbracht werden, indem Zusammenhänge zwischen den genannten Ressourcen und der standardisiert erfassten inklusiven Selbstwirksamkeit geprüft werden. Komplexe Zusammenhänge zwischen Ressourcen, Anforderungen und Beanspruchung sowie deren Auswirkungen auf den Unterricht in inklusiven Settings werden in diesem Beitrag nicht geprüft.

Folgende Fragestellungen stehen im Mittelpunkt:

- Welche Ressourcen nennen die befragten Grundschulehrkräfte, um mit Anforderungen in ihrem inklusiven Setting umzugehen?

- Welche Zusammenhänge gibt es zwischen diesen Ressourcen und in welchem Ausmaß erklärt die Ressourcennennung Unterschiede in der inklusiven Selbstwirksamkeit?

\section{Methode}

\subsection{Stichprobe und Untersuchungsdesign}

Zur Beantwortung der Forschungsfragen wurden Daten aus dem Forschungsprojekt BISU (Belastungen in einer inklusiven Schule und im gemeinsamen Unterricht 
Tab. 1 Stichprobe

\begin{tabular}{lllll}
\hline & $M$ & $S D$ & Min & Max \\
\hline Alter & 42,95 & 8,79 & 25 & 57 \\
Berufsjahre & 15,17 & 7,91 & 1 & 31 \\
Anteil an Kindern mit (sonder-)pädagogischem Bedarf & $48 \%$ & $27 \%$ & $5 \%$ & $100 \%$ \\
in der Klasse & & & & \\
\hline
\end{tabular}

begegnen) genutzt. Die Daten wurden in einem Mixed-Methods-Design sowohl qualitativ als auch quantitativ erhoben und ausgewertet. Dabei wurden im Sommer 2018 telefonische Leitfadeninterviews mit freiwilligen Grundschullehrkräften $(N=55)$ eines großstädtischen Schulamtsbezirkes in Bayern aus zehn Schulen mit einem teilweise hohen Anteil an Kindern mit (sonder-)pädagogischem Förderbedarf (s. Tab. 1) geführt. Im Anschluss an das Interview wurde den Lehrkräften ein Online-Fragebogen zugesandt. Die Analysestichprobe umfasst 43 Lehrkräfte, von denen vollständige Daten vorliegen.

\subsection{Instrumente und Variablen}

Der Interviewleitfaden enthält Fragen zu Anforderungen und Ressourcen in inklusiven Settings: Die Ressourcen wurden über eine erzählgenerierende Einstiegsfrage zum erfolgreichen Umgang mit Anforderungen in inklusiven Settings erhoben.

Tab. 2 Qualitatives Instrument - Ausschnitt des Interviewleitfadens zu Ressourcen

\begin{tabular}{ll}
\hline $\begin{array}{l}\text { Erzählgenerierende } \\
\text { Einstiegsfrage }\end{array}$ & Nachfragen \\
\hline Was tun Sie, um mit & Soziale Unterstützung: Wer hilft Ihnen da? \\
diesen Belastungen & Selbstregulation: Wie gehen Sie mit negativen Gefühlen und Stress um? \\
(belastende & Überzeugungen, Werthaltungen und motivationale Orientierungen: Welche \\
Situationen und Fälle) & Rolle spielen Ihre eigenen Einstellungen und Selbstwirksamkeitserwartungen? \\
klarzukommen? &
\end{tabular}

Tab. 3 Quantitatives Instrument - Adaption Bosse und Spörer (2014)

\begin{tabular}{|c|c|c|c|c|c|c|c|}
\hline Skala & Beispielitem & Items & Cron & $M$ & $S D$ & Min & $\operatorname{Max}$ \\
\hline $\begin{array}{l}\text { Gesamtskala: Selbs } \\
\text { Unterricht }\end{array}$ & wirksamkeit inklusiver & 8 & 0,78 & 2,68 & 0,49 & 1,25 & 3,88 \\
\hline $\begin{array}{l}\text { Selbstwirksamkeit } \\
\text { bezogen auf } \\
\text { die Gestaltung } \\
\text { inklusiven } \\
\text { Unterrichts }\end{array}$ & $\begin{array}{l}\text { Ich weiß, dass ich ein Un- } \\
\text { terrichtsthema so vielfältig } \\
\text { aufbereiten kann, dass } \\
\text { sogar Kinder mit sehr ho- } \\
\text { hem Förderbedarf aktiv } \\
\text { am Unterricht teilnehmen } \\
\text { können. }\end{array}$ & 4 & 0,78 & 2,46 & 0,62 & 1,00 & 4,00 \\
\hline $\begin{array}{l}\text { Selbstwirksamkeit } \\
\text { bezogen auf den } \\
\text { Umgang mit } \\
\text { Unterrichtsstö- } \\
\text { rungen }\end{array}$ & $\begin{array}{l}\text { Ich bin in der Lage, stö- } \\
\text { rendem Verhalten im } \\
\text { Klassenraum vorzubeugen, } \\
\text { bevor es auftritt. }\end{array}$ & 4 & 0,78 & 2,87 & 0,56 & 1,50 & 4,00 \\
\hline
\end{tabular}

4-stufige Likertskala; sprachliche Adaptionen kursiv 
In spezifischen Nachfragen wurde dabei insbesondere auf soziale Unterstützung, Selbstregulation, Überzeugungen und Werthaltungen sowie motivationale Orientierungen eingegangen (s. Tab. 2).

In einem Online-Fragebogen wurde flankierend die inklusive Selbstwirksamkeit anhand von zwei der drei Subskalen des KIESEL-Instruments (Bosse und Spörer 2014) erhoben, da die Items sich auf Selbstwirksamkeitserwartungen im Unterricht beziehen. Die Skalen wurden zudem sprachlich minimalinvasiv an ein weites Inklusionsverständnis angepasst (s. Tab. 3).

\subsection{Qualitative und quantitative Analysen}

In einem ersten Schritt erfolgte die qualitative Auswertung der Interviewdaten in Anlehnung an das Ablaufmodell der qualitativen Inhaltsanalyse nach Mayring (2015). Dabei wurden Prozesse der Strukturierung und Zusammenfassung miteinander verknüpft. Die Hauptkategorien zur Kodierung des Materials (Soziale Unterstützung, Selbstregulation, Überzeugungen und Werthaltungen, Motivationale Orientierungen) wurden zunächst deduktiv an das Material herangetragen, gebündelt und begrifflich angepasst sowie später durch eine induktiv gewonnene Hauptkategorie (Pädagogisch-didaktisches Wissen) ergänzt. Alle Hauptkategorien wurden inhaltlich durch induktiv gewonnene Subkategorien angereichert, sodass spezifische Informationen über die Ressourcen generiert werden konnten. Die Güte des Vorgehens wurde konsensuell und argumentativ validiert und über die Berechnung einer Intercoderübereinstimmung abgesichert $(0,80 \leq \kappa \leq 0,93)$.

Um in einem zweiten Schritt das angenommene Modell prüfen zu können, wurden die qualitativ gewonnenen Hauptkategorien für den weiteren Analyseprozess quantifiziert und z-transformiert sowie Korrelationen zwischen den vier qualitativ identifizierten Hauptkategorien und der quantitativ erhobenen inklusiven Selbstwirksamkeit ermittelt. Nach Prüfung der Voraussetzungen (keine Ausreißer; keine Autokorrelation: Durbon-Watson-Statistik 2,3; keine Multikollinearität: 0,99 $\leq$ Toleranz $\leq 1,0 ; \mathrm{Va}$ riationsinflationsfaktor $=1,0$ ) wurde mit einer schrittweisen multiplen Regression geprüft, inwiefern die Ressourcennennung die inklusive Selbstwirksamkeit vorhersagt.

\section{Ergebnisse}

\subsection{Ergebnisse der qualitativen Analysen}

Als hilfreiche Ressourcen im Umgang mit Anforderungen in inklusiven Settings konnten vier zentrale Hauptkategorien identifiziert werden (Forschungsfrage 1). Dabei nennen die Lehrkräfte die deduktiv gesetzten Hauptkategorien Soziale Unterstützung, Selbstregulation sowie Überzeugungen und Werthaltungen am häufigsten (s. Tab. 4). Die deduktiv angenommene Kategorie Motivationale Orientierungen ließ sich nicht mit einschlägigen Aussagen füllen. Insbesondere die Erwartung, dass in dieser Kategorie spezifische Selbstwirksamkeitserwartungen geäußert würden, konnte nicht bestätigt werden. Stattdessen beschrieben die Lehrkräfte allgemein ihr 
Tab. 4 Übersicht der Hauptkategorien (absteigend sortiert nach Häufigkeit)

\begin{tabular}{llllll}
\hline Ressourcen & $N$ & $M$ & $S D$ & Min & Max \\
\hline (1) Soziale Unterstützung & 223 & 5,19 & 3,73 & 0 & 20 \\
(2) Selbstregulation & 174 & 4,05 & 2,19 & 0 & 10 \\
(3) (Inklusive) Überzeugungen, Werthaltungen & 112 & 2,60 & 2,10 & 0 & 8 \\
und Vertrauen in die eigenen Fähigkeiten & & & & & \\
(4) Pädagogisch-didaktisches Wissen & 65 & 1,51 & 2,05 & 0 & 8 \\
Gesamt & 574 & 13,35 & 5,91 & 3 & 28 \\
\hline
\end{tabular}

Vertrauen in die eigenen Fähigkeiten, das als Subkategorie zusammengefasst und der Hauptkategorie Überzeugungen und Werthaltungen zugeordnet wurde (s. auch Kap. 6). Zusätzlich zu den deduktiv gefundenen Hauptkategorien wurde induktiv Pädagogisch-didaktisches Wissen als weitere Hauptkategorie identifiziert.

Neben Unterschieden in den Häufigkeiten der Hauptkategorien fallen große Unterschiede zwischen den Lehrkräften auf. Die Lehrkräfte nennen im Mittel rund 13 Ressourcen $(S D=5,91)$, wobei das Spektrum der Nennungen von sehr wenigen $(M i n=3)$ bis zu sehr vielen $($ Max $=28)$ reicht. 42 der 43 befragten Lehrkräfte tätigen Aussagen zu Ressourcen aus mindestens zwei Hauptkategorien, nur eine Lehrkraft beschreibt ausschließlich Ressourcen in der Hauptkategorie Selbstregulation. Differenzierte Auskunft über das Ressourcenspektrum der Lehrkräfte geben die nachfolgend berichteten und punktuell durch Ankerbeispiele illustrierten Subkategorien.

\subsubsection{Soziale Unterstützung (HK 1)}

Die Aussagen in der Kategorie Soziale Unterstützung beziehen sich nicht auf die Qualität oder Intensität wahrgenommener oder erlebter sozialer Unterstützung, sondern auf Quellen sozialer Unterstützung und wurden entlang genannter Akteurinnen und Akteure sowie Institutionen in sieben Subkategorien gebündelt. Eine Lehrkraft nennt soziale Unterstützung nicht als Ressource. Eine besonders wichtige Quelle der Unterstützung ist das Kollegium, das 41 der 43 Lehrkräfte nennen. In der zweiten Subkategorie wird von 31 Lehrkräften ein vielfältiges multiprofessionelles Netzwerk verschiedener Professionen und Institutionen (z. B. Sozial- und Sonderpädagogik, Hort) beschrieben. Von einigen Lehrkräften werden der Austausch und die Zusammenarbeit mit den Eltern $(n=15)$, das Aufsuchen von Beratungs- und Fortbildungsangeboten $(n=13)$ sowie der Austausch mit und die Unterstützung durch die Schulleitung $(n=12)$ geschildert. Sechs Lehrkräfte nennen ihr privates Umfeld als Unterstützungsquelle. Außerdem werden vereinzelt Schülerinnen und Schüler in der Klasse $(n=3)$ bei problematischen Situationen um Hilfe gebeten.

\subsubsection{Selbstregulation $(H K 2)$}

42 der 43 Lehrkräfte beschreiben als hilfreiche Ressource Strategien und Maßnahmen zum Erhalt der Erholungs- und Distanzierungsfähigkeit sowie der bewussten Regulation von Emotionen, welche zusammenfassend zu der Hauptkategorie Selbstregulation gebündelt werden. Die am häufigsten genannte Subkategorie ist 
Ausgleich und Auszeit (z.B. Erholungs- und Entspannungsstrategien) und wird von 31 Lehrkräften benannt. Knapp die Hälfte der Lehrkräfte nennt auch die eigene Distanzierungsfähigkeit als Ressource: „Ich bearbeite [...] alles Schulische in der Schule. [...] Aber mir hilft das, dass das Schulische weitestgehend in der Schule bleibt“" (LK 37). Auch Strategien und Maßnahmen zur Emotionsregulation werden von 16 Lehrkräften beschrieben. Mit Blick auf die Emotionsregulation ist auch die Subkategorie Unlösbarkeiten und Grenzen erkennen für 15 Lehrkräfte bedeutsam. Im Rahmen von Unlösbarkeiten sprechen die Lehrkräfte darüber, gezielt Abstriche zu machen und Unlösbarkeiten zu akzeptieren. Eine weitere Subkategorie bezieht sich auf die Reflexion des eigenen Handelns oder der beanspruchenden Situation sowie deren positive Relativierung $(n=14)$. Drei Lehrkräfte beschreiben auch, gezielt auf die eigene Gesundheit zu achten.

\subsection{3 (Inklusive) Überzeugungen, Werthaltungen und Vertrauen in die eigenen Fähigkeiten (HK 3)}

Auf die Nachfrage nach der Rolle der persönlichen Einstellungen und Selbstwirksamkeitserwartungen beschreiben 36 Lehrkräfte theoretisch einschlägiges Gedankengut zur Hauptkategorie (Inklusive) Überzeugungen, Werthaltungen und Vertrauen in die eigenen Fähigkeiten. 25 Lehrkräfte geben optimistische inklusive Überzeugungen wieder, indem sie sich selbst als inklusionsorientierte Menschen beschreiben, die Inklusion trotz Chancen und Grenzen grundsätzlich befürworten: ,[...] Ich finde das inklusive Arbeiten hochinteressant und ich glaube, dass das für viele Inklusionskinder was ganz Tolles ist und auch der Normschüler hat was davon“ (LK 22). Weitere Überzeugungen betreffen das pädagogische Ethos, alle Kinder bestmöglich zu fördern $(n=9)$ sowie allgemeine positive Einstellungen zum Beruf $(n=4)$. Weniger Subkategorien konnten im Bereich der Werthaltungen identifiziert werden. Neben der Werthaltung, alle Kinder akzeptieren und wertschätzen $(n=12) \mathrm{zu}$ wollen, beschreiben sieben Lehrkräfte auch Fürsorge und Gerechtigkeit gegenüber allen Kindern. Trotz direkter Nachfrage äußert keine Lehrkraft spezifische (inklusive) Selbstwirksamkeitserwartungen. 13 Lehrkräfte beschreiben stattdessen allgemein das Vertrauen in die eigenen Fähigkeiten als wichtige Ressource, ohne dass die Fähigkeiten von ihnen näher spezifiziert werden: „Also ohne meine Fähigkeiten, wenn ich mir da nicht vertrauen würde, dann wäre es vorbei“" (LK 52).

\subsubsection{Pädagogisch-didaktisches Wissen (HK 4)}

Als impliziter Ausdruck pädagogisch-didaktischen Wissens schreiben 24 der 43 Lehrkräfte pädagogischen sowie didaktischen Strategien ein Entlastungspotenzial zu. Während als Reaktion auf Störungen $(n=13)$ oftmals Sanktionen geschildert werden, zielen andere Subkategorien insgesamt eher auf eine Verbesserung des Unterrichts, der Unterrichtsqualität sowie der persönlichen Beziehung zum Kind. Dabei werden Differenzierungsmaßnahmen und eine offene Unterrichtsorganisation sowie Reflexionen und Gespräche mit dem Kind von acht Lehrkräften genannt, während fünf Lehrkräfte auch beschreiben, Lösungsmöglichkeiten zu suchen und anzuwenden sowie Unterricht zu rhythmisieren und auf Rituale zu achten. Drei Lehrkräfte finden 
Tab. 5 Interkorrelationen der Ressourcen (z-Faktorwert) und der inklusiven Selbstwirksamkeit

\begin{tabular}{llllc}
\hline & $(1)$ & $(2)$ & $(3)$ & (4) \\
\hline (1) Soziale Unterstützung & - & - & - & - \\
(2) Selbstregulation & $-0,01$ & - & - & - \\
(3) (Inklusive) Überzeugungen, Werthaltungen und & 0,21 & 0,22 & - & - \\
Vertrauen in die eigenen Fähigkeiten & & & & - \\
(4) Pädagogisch-didaktisches Wissen & 0,22 & 0,07 & 0,09 & - \\
(5) Selbstwirksamkeit inklusiver Unterricht & $0,31^{*}$ & $0,36^{* *}$ & $0,29 *$ & $-0,07$ \\
\hline
\end{tabular}

$* p<0,05$ (1-seitig), **p $<0,01$ (1-seitig)

es entlastend, wenn sie Kinder positiv bestärken und die Beziehung pflegen. Darüber hinaus nennen zwei Lehrkräfte die Gemeinsame Zielvereinbarung mit dem Kind. Das pädagogisch-didaktische Wissen wird sowohl allgemein als auch spezifisch im Umgang mit als belastend erlebten Situationen und Fällen beschrieben.

\subsection{Ergebnisse der quantitativen Analysen}

Hinsichtlich der Frage nach Zusammenhängen zwischen den identifizierten Ressourcen und der inklusiven Selbstwirksamkeit (Forschungsfrage 2) gibt zunächst Tab. 5 Auskunft: Es finden sich keine Zusammenhänge zwischen den qualitativ erfassten, quantifizierten Ressourcenkategorien. Die inklusive Selbstwirksamkeit hängt signifikant mit den drei deduktiv gesetzten Hauptkategorien zusammen, nicht jedoch mit der induktiv gefundenen Hauptkategorie.

Angesichts der gefundenen Korrelationen gingen nur die drei deduktiv gesetzten Hauptkategorien als Prädiktoren in die schrittweise regressionsanalytische Vorhersage der inklusiven Selbstwirksamkeit ein. Die Hauptkategorien Soziale Unterstützung $(\beta=36, p<0,05)$ und Selbstregulation $(\beta=0,35, p<0,05)$ erklären gemeinsam $20 \%$ der Varianz in der inklusiven Selbstwirksamkeit $(F(2,38)=5,88, p<0,01)$. Die Hauptkategorie (Inklusive) Überzeugungen, Werthaltungen und Vertrauen in die eigenen Fähigkeiten verfehlt im multivariaten Modell das Aufnahmekriterium $(p<0,10)$ eines signifikanten Erklärungsbeitrags und wird daher aus dem Modell ausgeschlossen. Aufgrund fehlender Korrelationen mit inklusiver Selbstwirksamkeit wurden Kontextinformationen, Berufsjahre und Förderanteil, zunächst nicht in das Modell aufgenommen. In weiteren Analysen konnten unter Hinzunahme dieser beiden Kontrollvariablen keine substantiellen Veränderungen in der Varianzaufklärung gefunden werden.

\section{Zusammenfassung und Diskussion}

In Übereinstimmung mit bereits vorliegenden Studien zu Arbeitsressourcen von Lehrkräften (Bennemann 2019; Bosse et al. 2016; Döring-Seipel und Dauber 2010; Schaarschmidt 2005) beschreiben die befragten Grundschullehrkräfte für den Umgang mit Anforderungen in inklusiven Setting am häufigsten soziale Unterstützung gefolgt von Selbstregulation. Inklusive Überzeugungen, Werthaltungen und das Vertrauen in die eigenen Fähigkeiten rangieren erst an dritter Stelle. Spezifische inklu- 
sive Selbstwirksamkeitserwartungen werden überraschenderweise nicht geäußert, obwohl die Lehrkräfte direkt nach diesen gefragt wurden. Stattdessen beschreiben die Lehrkräfte eher allgemein die Bedeutsamkeit des Vertrauens in ihre eigenen Fähigkeiten, ohne auf ihren Umgang mit speziellen Anforderungen in inklusiven Settings, wie eine adaptive Unterrichtsgestaltung oder den eigenen Umgang mit störendem Verhalten (Bosse und Spörer 2014), einzugehen. Auch wenn die Lehrkräfte nicht explizit nach ihrem allgemeinen pädagogischen Wissen gefragt wurden, konnte als weitere Ressource pädagogisch-didaktisches Wissen identifiziert werden. Dabei könnte gerade das Wissen um Unterrichtsmethoden, Leistungsbeurteilung, individuelle Lernprozesse und individuelle Besonderheiten von Kindern (Voss et al. 2015) eine wichtige Basis sein, um die von Lindner und Schwab (2020) identifizierten pädagogischen Tätigkeiten in inklusiven Settings (s. Abschn. 2.1) erfolgreich umsetzen zu können. Studien bestätigen, dass insbesondere bei angehenden Lehrkräften das Wissen um den Umgang mit Unterrichtsstörungen mit einem günstigen Beanspruchungserleben einhergeht (Dicke et al. 2015) und eine positive Beziehung zwischen Lehrkräften sowie Schülerinnen und Schülern für das Wohlbefinden der Lehrkräfte zuträglich ist (Aldrup et al. 2018).

$\mathrm{Zu}$ diskutieren ist die Zuordnung der Subkategorie Vertrauen in die eigenen Fähigkeiten: Selbstbezogene Kognitionen können nach Baumert und Kunter (2011) sowohl den Werthaltungen und Überzeugungen als auch den motivationalen Orientierungen zugeordnet werden, wobei Selbstwirksamkeitserwartungen als motivationale Orientierungen im Kontext der Belastungs- und Beanspruchungsforschung teilweise auch gemeinsam mit der Selbstregulation geführt werden (Abele und Candova 2007; Rothland 2013a). Da die gefundenen Aussagen der Lehrkräfte weder auf die Regulation von Emotionen oder den Erhalt der eigenen Energie gerichtet sind noch explizit motivationale Tendenzen erkennen lassen, sondern das allgemeine Vertrauen der Lehrkräfte in die eigenen Fähigkeiten beschreiben, wurden sie hier gemeinsam mit Überzeugungen und Werthaltungen gebündelt. Von der quantitativ erfassten inklusiven Selbstwirksamkeit lassen sich die qualitativ erfassten Aussagen zum Vertrauen der Lehrkräfte in die eigenen Fähigkeiten durch deren Allgemeinheit und fehlenden Inklusionsbezug abgrenzen.

Ein weiteres Ergebnis der vorliegenden Untersuchung zeigt signifikante Zusammenhänge zwischen sozialer Unterstützung, Selbstregulation sowie Überzeugungen, Werthaltungen und Vertrauen in die eigenen Fähigkeiten mit der inklusiven Selbstwirksamkeit. Die inklusive Selbstwirksamkeit korreliert allerdings nicht mit dem pädagogisch-didaktischen Wissen. Für die weiteren Analysen wurde die inklusive Selbstwirksamkeit als abhängige Variable betrachtet, da sie Hinweise auf unterrichtliches Handeln geben kann. Eine intensivere Nutzung eines umfangreichen (multi-)professionellen Netzwerkes (soziale Unterstützung) sowie ein höheres Ausmaß an Erholungs- und Distanzierungsfähigkeit und Emotionsregulation (Selbstregulation) sagen in dieser Untersuchung höhere inklusive Selbstwirksamkeit der Lehrkräfte vorher. Hingegen erweisen sich die von den Lehrkräften berichteten Überzeugungen, Werthaltungen und das Vertrauen in die eigenen Fähigkeiten für die inklusive Selbstwirksamkeit als nicht bedeutsam. Mit Blick auf die theoretische Verortung muss überlegt werden, inwiefern die Hauptkategorie Überzeugungen, Werthaltungen und Vertrauen in die eigenen Fähigkeiten sowie die Selbstre- 
gulation (Prädiktor) die inklusive Selbstwirksamkeit (Kriterium) implizieren. Das Kompetenzmodell von COAKTIV legt nahe, dass diese Kompetenzaspekte eng miteinander verknüpft sind, was auch die unterschiedliche theoretische Verortung von Selbstwirksamkeitserwartungen verdeutlicht.

\section{Limitationen und Ausblick}

Der gleichzeitige Zugriff auf qualitative und quantitative Daten erlaubt zum einen, personinterne und personexterne Ressourcenbereiche für einen erfolgreichen Umgang mit Anforderungen umfassend zu erheben, und zum anderen, Zusammenhänge zu weiteren standardisierten Variablen, wie der inklusiven Selbstwirksamkeit, zu prüfen. Obwohl Selbstregulation in enger Verbindung mit Überzeugungen, Werthaltungen und dem Vertrauen in die eigenen Fähigkeiten steht und beide Ressourcen oftmals insgesamt unter dem Begriff personale Ressourcen geführt werden (Döring-Seipel und Dauber 2010), sprechen die hier berichteten Ergebnisse deutlich für eine differenziertere Betrachtung, da sie unterschiedliche Beiträge in der Varianzaufklärung der inklusiven Selbstwirksamkeit leisten. Dennoch bleibt ein Großteil der Varianz unaufgeklärt, was die Frage nach zusätzlichen relevanten Größen (z. B. weitere Personen- oder Kontextmerkmale auf Schul- und Klassenebene) aufwirft. Auch die Nachfragen im Interviewleitfaden (s. Tab. 2) sind zu diskutieren. Während gezielt nach sozialer Unterstützung, Selbstregulation und Überzeugungen, Werthaltungen und Selbstwirksamkeit gefragt wurde, wurde das pädagogisch-didaktische Wissen ohne spezielle Nachfragen von den Lehrkräften in den Interviews produziert. Mögliche Zusammenhänge zwischen dem pädagogisch-didaktischen Wissen und der inklusiven Selbstwirksamkeit könnten daher unentdeckt bleiben, die sich bei expliziter Nachfrage oder zusätzlicher Gewichtung der freien Äußerungen der Lehrkräfte zeigen könnten. Zudem ist unklar, ob der Begriff Selbstwirksamkeit alltagssprachlich von den Lehrkräften ausreichend verstanden und genutzt wird. Da Arbeitsressourcen individuell bedeutsam sind und das subjektive Erleben belastender Fallmerkmale und Situationen als Anforderung in inklusiven Settings zwischen den Lehrkräften variieren kann, ist auch die Anwendung, Nutzung und Wirkung verschiedener Ressourcen zwischen den Lehrkräften und je nach Fall und Situation unterschiedlich und kann zudem durch weitere Kontextfaktoren bestimmt sein (Bakker und Demerouti 2007). Das bedeutet, dass nicht per se jede Lehrkraft alle hier identifizierten Ressourcen als hilfreich im Umgang mit ihren spezifischen Anforderungen in inklusiven Settings erachtet. Dies unterstreicht der Diskurs um soziale Unterstützung: Soziale Unterstützung kann zwar eine gewinnbringende Ressource sein, die Quellen der sozialen Unterstützung müssen hierzu aber auch passende Formen der Unterstützung für die jeweiligen Anforderungen anbieten (Kienle et al. 2006). In dieser Untersuchung bleiben die kausale Bedeutung der Ressourcen für unterrichtliches Handeln sowie die Prüfung günstiger Effekte auf das Beanspruchungserleben der Lehrkräfte offen und müssten in einem Längsschnitt überprüft werden. Ungeklärt bleibt auch, inwiefern sich die inklusive Selbstwirksamkeit auf das unterrichtliche Handeln in der Praxis niederschlägt. Eine solche Prüfung wäre evident, da Selbstwirksamkeit selbst als personinterne Ressource fungieren kann. 
Insgesamt können die qualitativen Befunde Lehrkräfte dazu anregen, ihre Ressourcenprofile zu überdenken, zu revidieren oder anzureichern. Wenngleich der Umgang mit Anforderungen und ihre Bewertung individuell ist, kann bereits präventiv ein ressourcenorientiertes Empowerment bislang generell oder individuell unterrepräsentierte Ressourcen von Lehrkräften aufdecken und stärken helfen. Die quantitativen Befunde weisen hier speziell auf die Bedeutung eines sozialen Netzwerkes und emotional-energetischer Selbstregulation hin, da diese Ressourcen eine höhere inklusive Selbstwirksamkeit vorhersagen. Die Ergebnisse geben somit Hinweise für Ansatzpunkte in der Aus-, Fort- und Weiterbildung von Lehrkräften. Diese ersten Implikationen sowie eine mögliche Priorisierung der Relevanz der Ressourcen (für Selbstwirksamkeit, unterrichtliches Handeln und Belastungsmanagement) müssten jedoch in weiteren (Interventions-)Studien geprüft werden.

Funding Open Access funding enabled and organized by Projekt DEAL.

Open Access Dieser Artikel wird unter der Creative Commons Namensnennung 4.0 International Lizenz veröffentlicht, welche die Nutzung, Vervielfältigung, Bearbeitung, Verbreitung und Wiedergabe in jeglichem Medium und Format erlaubt, sofern Sie den/die ursprünglichen Autor(en) und die Quelle ordnungsgemäß nennen, einen Link zur Creative Commons Lizenz beifügen und angeben, ob Änderungen vorgenommen wurden.

Die in diesem Artikel enthaltenen Bilder und sonstiges Drittmaterial unterliegen ebenfalls der genannten Creative Commons Lizenz, sofern sich aus der Abbildungslegende nichts anderes ergibt. Sofern das betreffende Material nicht unter der genannten Creative Commons Lizenz steht und die betreffende Handlung nicht nach gesetzlichen Vorschriften erlaubt ist, ist für die oben aufgeführten Weiterverwendungen des Materials die Einwilligung des jeweiligen Rechteinhabers einzuholen.

Weitere Details zur Lizenz entnehmen Sie bitte der Lizenzinformation auf http://creativecommons.org/ licenses/by/4.0/deed.de.

\section{Literatur}

Abele, A.E., \& Candova, A. (2007). Prädikatoren des Belastungserlebens im Lehrerberuf. Befunde einer 4-jährigen Längsschnittstudie. Zeitschrift für Pädagogik, 21(2), 107-118.

Aldrup, K., Klusmann, U., Lüdtke, O., Göllner, R., \& Trautwein, U. (2018). Student misbehavior and teacher well-being: testing the mediating role of the teacher-student relationship. Learning and Instruction, 58, 126-136.

Bakker, A.B., \& Demerouti, E. (2007). The job demands-resources model: state of the art. Journal of Managerial Psychology, 22(3), 309-328.

Bandura, A. (1977). Self-efficacy: toward a unifying theory of behavioral change. Psychological Review, 84(2), 191-215.

Baumert, J., \& Kunter, M. (2011). Das Kompetenzmodell von COACTIV. In M. Kunter, J. Baumert \& W. Blum (Hrsg.), Professionelle Kompetenz von Lehrkräften. Ergebnisse des Forschungsprogramms COACTIV (S. 29-53). Münster: Waxmann.

Bennemann, E.-M. (2019). Die Arbeits- und Gesundheitssituation von Lehrkräften. Aufgaben, Belastungen und Ressourcen an integrierten und inklusiven Schulen. Münster: Waxmann.

Bosse, S., \& Spörer, N. (2014). Erfassung der Einstellung und der Selbstwirksamkeit von Lehramtsstudierenden zum inklusiven Unterricht. Empirische Sonderpädagogik, 4, 279-299.

Bosse, S., Henke, T., Jäntsch, C., Lambrecht, J., Vock, M., \& Spörer, N. (2016). Die Entwicklung der Einstellung zum inklusiven Lernen und der Selbstwirksamkeit von Grundschullehrkräften. Empirische Sonderpädagogik, 1, 103-116. 
Bosse, S., Jäntsch, C., Henke, T., Lambrecht, J., Koch, H., \& Spörer, N. (2017). Das Zusammenspiel der Offenheit für Innovationen, der Einstellung zum inklusiven Lernen und der Selbstwirksamkeit von Lehrkräften. Zeitschrift für Bildungsforschung, 7(2), 131-146.

Cramer, C., Friedrich, A., \& Merk, S. (2018). Belastung und Beanspruchung im Lehrerinnen- und Lehrerberuf: Übersicht zu Theorien, Variablen und Ergebnissen in einem integrativen Rahmenmodell. Bildungsforschung, 15(1), 1-23.

Dicke, T., Parker, P. D., Holzberger, D., Kunina-Habenicht, O., Kunter, M., \& Leutner, D. (2015). Beginning teachers' efficacy and emotional exhaustion: latent changes, reciprocity, and the influence of professional knowledge. Contemporary Educational Psychology, 41, 569-583.

Dicke, T., Stebner, F., Linninger, C., Kunter, M., \& Leutner, D. (2018). A longitudinal study of teachers' occupational well-being: applying the job demands-resources model. Journal of Occupational Health Psychology, 23(2), 262-277.

Döring-Seipel, E., \& Dauber, H. (2010). Was hält Lehrer und Lehrerinnen gesund - die Bedeutung von Ressourcen, subjektiver Bewertung und Verarbeitung von Belastung für die Gesundheit von Lehrern und Lehrerinen. Schulpädagogik heute, 1(2), 1-16.

Elting, C., Kopp, B., \& Martschinke, S. (2019). Soziale Kompetenz und Integration von Grundschulkindern mit besonderem pädagogischen Förderbedarf. Erste Ergebnisse aus der KOMENSKI-Studie. In C. Donie, F. Foerster \& M. Obermayr (Hrsg.), Grundschulpädagogik zwischen Wissenschaft und Transfer (S. 296-302). Wiesbaden: Springer VS.

Fauth, B., Decristan, J., Decker, A.-T., Büttner, G., Hardy, I., Klieme, E., \& Kunter, M. (2019). The effects of teacher competence on student outcomes in elementary science education: the mediating role of teaching quality. Teaching and Teacher Education, 86, 1-14.

Hecht, P., Niedermair, C., \& Feyerer, E. (2016). Einstellungen und inklusionsbezogene Selbstwirksamkeitsüberzeugungen von Lehramtsstudierenden und Lehrpersonen im Berufseinstieg - Messverfahren und Befunde aus einem Mixed-Methods-Design. Empirische Sonderpädagogik, 8(1), 86-102.

Hedderich, I. (2016). Lehrergesundheit im Kontext schulischer Inklusion. Prävention und Gesundheitsförderung, 11(1), 34-39.

Kiel, E., Braun, A., Muckenthaler, M., Heimlich, U., \& Weiss, S. (2020). Self-efficacy of teachers in inclusive classes. How do teachers with different self-efficacy beliefs differ in implementing inclusion? European Journal of Special Needs Education, 35(3), 333-349.

Kienle, R., Knoll, N., \& Renneberg, B. (2006). Soziale Ressourcen und Gesundheit: soziale Unterstützung und dyadisches Bewältigen. In B. Renneberg \& P. Hammelstein (Hrsg.), Gesundheitspsychologie (S. 107-122). Berlin, Heidelberg, New York: Springer.

Klassen, R.M., \& Tze, V.M. (2014). Teachers' self-efficacy, personality, and teaching effectiveness: a meta-analysis. Educational Research Review, 12, 59-76.

Klusmann, U., \& Richter, D. (2014). Beanspruchungserleben von Lehrkräften und Schülern in der Primarstufe. Zeitschrift für Pädagogik, 60(2), 202-224.

Knigge, M., \& Rotter, C. (2015). Unterrichtsplanungen bei Lehramtsstudierenden im Falle der Wahrnehmung von vermeintlich ,,besonderen“ Schülerinnen und Schülern und ihr Zusammenhang mit Selbstwirksamkeitsüberzeugungen und Einstellungen in Bezug zu Inklusion - beispielhafte Mixed-MethodAnalysen aus der EiLInk-Studie. Empirische Sonderpädagogik, 3, 223-240.

König, J., Gerhard, K., Kaspar, K., \& Melzer, C. (2019). Professionelles Wissen von Lehrkräften zur Inklusion: Überlegungen zur Modellierung und Erfassung mithilfe standardisierter Testinstrumente. Pädagogische Rundschau, 73(1), 43-64.

Krause, A., Dorsemagen, C., \& Baeriswyl, S. (2013). Zur Arbeitssituation von Lehrerinnen und Lehrern: Ein Einstieg in die Lehrerbelastungs und -gesundheitsforschung. In M. Rothland (Hrsg.), Belastung und Beanspruchung im Lehrerberuf (S. 61-80). Wiesbaden: VS.

Kyriacou, C. (2001). Teacher stress: directions for future research. Educational Review, 53(1), $27-35$.

Lehr, D. (2014). Belastung und Beanspruchung im Lehrerberuf. Gesundheitliche Situation und Evidenz für Risikofaktoren. In E. Terhart, H. Bennewitz \& M. Rothland (Hrsg.), Handbuch der Forschung zum Lehrerberuf (2. Aufl. S. 947-967). Münster: Waxmann.

Lindner, K.-T., \& Schwab, S. (2020). Differentiation and individualisation in inclusive education: a systematic review and narrative synthesis. International Journal of Inclusive Education, 1-21. https://doi. org/10.1080/13603116.2020.1813450.

Lübke, L., Meyer, J., \& Christiansen, H. (2016). Effekte von Einstellungen und subjektiven Erwartungen von Lehrkräften: Die Theorie des geplanten Verhaltens im Rahmen schulischer Inklusion. Empirische Sonderpädagogik, 8(3), 225-238. 
Martschinke, S., Elting, C., Grüning, M., Kopp, B., Niessen, C., \& Schröder, C. (2020). Belastende Fälle in inklusiven Settings - erste Ergebnisse aus dem Kooperationsprojekt BISU. In N. Skorsetz, M. Bonanati \& D. Kucharz (Hrsg.), Diversität und soziale Ungleichheit (S. 277-281). Wiesbaden: Springer.

Mayring, P. (2015). Qualitative Inhaltsanalyse. Grundlagen und Techniken. Weinheim, Basel: Beltz.

Peperkorn, M., Müller, K., Mertz, K., \& Paulus, P. (2020). Dealing with inclusion - teachers' assessment of internal and external resources. International Journal of Inclusive Education, 1-17. https://doi.org/ $10.1007 / \mathrm{s} 11553-018-0660-3$.

Rothland, M. (2013a). Belastung und Beanspruchung im Lehrerberuf und die Modellierung professioneller Kompetenz von Lehrerinnen und Lehrern. In M. Rothland (Hrsg.), Belastung und Beanspruchung im Lehrerberuf (S. 2-20). Wiesbaden: VS.

Rothland, M. (2013b). Soziale Unterstützung im. In M. Rothland (Hrsg.), Belastung und Beanspruchung im Lehrerberuf (S. 231-250). Wiesbaden: VS.

Rothland, M., \& Klusmann, U. (2016). Belastung und Beanspruchung im Lehrerberuf. In M. Rothland (Hrsg.), Beruf Lehrer/Lehrerin. Ein Studienbuch (S. 351-369). Münster: Waxmann.

Rudow, B. (1994). Die Arbeit des Lehrers. Zur Psychologie der Lehrertätigkeit, Lehrerbelastung und Lehrergesundheit (1. Aufl.). Bern: Huber.

Saloviita, T., \& Takala, M. (2010). Frequency of co-teaching in different teacher categories. European Journal of Special Needs Education, 25(4), 389-396.

Schaarschmidt, U. (2005). Halbtagsjobber? Psychische Gesundheit im Lehrerberuf - Analyse eines veränderungsbedürftigen Zustandes. Weinheim: Beltz.

Schaarschmidt, U., \& Kieschke, U. (2013). Beanspruchungsmuster im Lehrerberuf. In M. Rothland (Hrsg.), Belastung und Beanspruchung im Lehrerberuf (S. 81-98). Wiesbaden: VS.

Schwarzer, R., \& Warner, L.-M. (2014). Forschung zur Selbstwirksamkeit bei Lehrerinnen und Lehrern. In E. Terhart, H. Bennewitz \& M. Rothland (Hrsg.), Handbuch der Forschung zum Lehrerberuf (S. 662-678). Münster: Waxmann.

Stiller, M. (2015). Belastungen, Ressourcen und Beanspruchungen bei Lehrkräften. Bad Heilbrunn: Klinkhardt.

Urton, K., Wilbert, J., \& Hennemann, T. (2014). Der Zusammenhang zwischen der Einstellung zur Integration und der Selbstwirksamkeit von Schulleitungen und deren Kollegien. Empirische Sonderpädagogik, 1, 3-16.

Voss, T., Kunina-Habenicht, O., Hoehne, V., \& Kunter, M. (2015). Stichwort Pädagogisches Wissen von Lehrkräften: Empirische Zugänge und Befunde. Zeitschrift für Erziehungswissenschaft, 18(2), 187-223.

Wesselborg, B., Reiber, K., Richey, P., \& Bohl, T. (2014). Untersuchung der Lehrergesundheit im Mixed-Methods-Design unter Verwendung von Videografie. Lehrerbildung auf dem Prüfstand, 7(2), 157-174.

Wudy, D.-T., \& Jerusalem, M. (2011). Die Entwicklung von Selbstwirksamkeit und Belastungserleben bei Lehrkräften. Psychologie in Erziehung und Unterricht, 58(4), 254-267. 